\title{
TITLE: BALANCING ENERGY TO ESTIMATE DAMPING PARAMETERS IN FORCED OSCILLATORS
}

Jin-Wei Liang,

Department of Mechanical Engineering, MingChi University of Technology, 84 Gung-Juan Rd., Taishan, Taipei County, Taiwan, 24306, Republic of China. liangj@,ccsun.mit.edu.tw

Brian F. Feeny, Department of Mechanical Engineering, Michigan State University, 2328C, Engineering Bldg., East Lansing, MI 48824, USA.

feeny@egr.msu.edu

\section{ABSTRACT}

This study makes use of an energy balance to identify damping parameters in mechanical vibration systems. By balancing the energy input as registered in the force-displacement relationship of the real system against the energy lost theoretically in a damping model with unknown parameters, the identification algorithms are developed. We apply the estimation equations to both numerical and experimental systems, modeled with Coulomb plus viscous damping, at resonance to show the effectiveness and reliability of the new identification method. The equivalent viscous and dry-friction damping estimates obtained from the experimental system are compared to those obtained from the forced-resonance method to show their consistencies.

\section{INTRODUCTION}

Friction parameter estimation is based on the analysis of measured input and output responses. Our interest is in identifying parameters of basic friction models by making use of vibration properties. To this end, free vibration decrements have been exploited for systems with linear stiffness elements and "small" damping. Here, "small" damping means two things: the free response has sufficiently many extreme excursions that can be measured with good resolution; and the frequency of damped oscillation is approximately equal to the undamped natural frequency $\omega_{n}$. The classic logarithmic decrement scheme for viscous friction identification goes back to Helmholtz [1] and Rayleigh [2], while the constant decrement [3] can be used for estimation of Coulomb damping.

Jacobsen and Ayre [4] developed an approximate scheme for estimating both viscous and dry friction quantities from the free-vibration decrements by noting that the viscous friction dominates in the largeamplitude responses, and that Coulomb friction dominates in the small-amplitude oscillations. An exact formulation for the simultaneous estimation of Coulomb and viscous friction in oscillators has since been derived [5-7]. 
Free vibration decrements are not applicable if the damping is strong enough to preempt sufficient oscillations. As such, it makes sense to develop schemes for identifying friction parameters in forced oscillators. For instance, Stanway et al. [8] proposed identifying Coulomb and viscous damping parameters with a nonlinear least-squares scheme, which involves the on-line solution of several additional differential equations. Yao et al. [9] identified the Coulomb and viscous friction parameters by applying a recursive nonlinear least-squares algorithm. Chen and Tomlinson [10] proposed estimating damping parameters in nonlinear oscillators by utilizing the acceleration, velocity and displacement output and formulating the output in terms of series of frequency response functions. Tomlinson and Hibbert [11] applied the power dissipation to estimate Coulomb and hysteretic damping coefficients. Tomlinson [12] also measured the distortions in the complex receptance plots to identify damping parameters. Iourtchenko and Dimentberg [13] used stochastic averaging to identify nonlinear damping inprocess when the excitation was random. Iourtchenko et. al. [14], based on Dimentberg [15], applied a harmonic balance analysis to generate identification equations.

A scheme for extracting Coulomb and viscous friction parameters from forced oscillations based on the analytical solutions of Den Hartog [16] and Hundal [17] for the nonsticking response to harmonic excitations was formulated in our previous work [18]. We proposed an approximate forced-identification algorithm denoted as the analytical forced-resonance method. The analytical forced-resonance method employs an assumption of small viscous damping of the real system leading to a linear relationship between amplitudes of harmonic input and output motion. Analytical, numerical and experimental studies have shown the analytical forced-resonance method to be effective.

The limitations of the analytical forced-resonance method are that it is not applicable for damping which is not "small," it relies on analytical solutions of single-degree-of-freedom linear systems, and it does not treat friction models other than Coulomb plus viscous (see for example, references [19-24]). The need for analytical solutions can limit the identification of more complicated systems. Nonetheless, since the analytical forced-resonance method is based on an analytical solution, it can also serve as a benchmark in the continuing development of damping identification schemes for more general systems, for which analytical solutions are not available.

In this paper, we propose an energy balance as an alternative to analytical solutions for identifying friction parameters. We apply the energy-balance formulation to systems with co-existing Coulomb and viscous friction as an example. There are other more advanced friction models that can capture complicated friction dynamics, such as the work of Soom and co-workers [25], the state-variable models of Rice [26], Deterich [27], and Ruina [28], the bristle models of Hassig and Friedland [24] and Canudas de Wit et al. [29], the normal vibration models of Tolstoi [30], Oden and Martins [21] and Dankowicwz [31], and the contact compliance models [22-24, 32-35].

In developing the energy-balance method, the energy input of a physical system is registered in the force-displacement relationship. This energy input is balanced against the energy loss of a theoretical model, consisting of viscous damping and dry friction components. From this balance of energy, the estimation equations are derived, and applied to numerical and experimental systems in order to examine the reliabilities of the new identification method.

\section{THE ENERGY-DISSIPATION IDENTIFICATION}

The energy-dissipation identification method involves the balance between the energy dissipated by the friction force against the energy input to the system. This balance results in the "equivalent viscous and Coulomb damping" parameters, reminiscent to the traditional "equivalent viscous damping" concept seen in undergraduate textbooks, such as [36]. In the traditional approach, an equivalent viscous model replaces another nonlinear damping model such that there is an energy balance. The measured response amplitude and the nonlinear damping model are used together with a harmonic response assumption to 
compute the equivalent viscous damping coefficient. In this work, we suggest balancing energy using measured inputs and outputs to identify the coefficients of an assumed nonlinear damping model.

Consider a damped-forced oscillator with the following equation of motion:

$$
m \ddot{x}+k x+F(x, \dot{x})=a(t)
$$

where $m$ is the mass, $k$ is the stiffness, $x$ is the displacement, dots represent derivatives with respect to time, and $a(t)$ is the excitation. Multiplying (1) by $d x$ and integrating along the motion path $C$ yields the following energy-balance equation

$$
\int_{C}\{m \ddot{x}+k x+F(x, \dot{x})\} d x=\int_{C} a(t) d x .
$$

To assist the implementation of integration, the integration variable can be changed to time, thus

$$
\int_{t}^{t+T_{1}}\{m \ddot{x}+k x+F(x, \dot{x})\} \dot{x} d t=\int_{t}^{t+T_{1}} a(t) \dot{x} d t
$$

Here $T_{1}$ indicates a finite time interval. We define

$$
W_{d}=\int_{t}^{t+T_{1}} F(x, \dot{x}) \dot{x} d t ; \quad W_{a}=\int_{t}^{t+T_{1}} a(t) \dot{x} d t ; \quad W_{e}=\int_{t}^{t+T_{1}}\{m \ddot{x}+k x\} \dot{x} d t,
$$

so that equation (1) can be expressed

$$
W_{d}=W_{a}-W_{e}
$$

representing a balance between the dissipated, applied energy, and the sum of the kinetic and elastic energy, respectively. The terms in Eq. (2)can be quantified if $x(t)$ (and its derivatives) and $a(t)$ are measured. Then, by integrating and balancing both sides of Eq. (2) while expressing $F(x, \dot{x})$ using an assumed friction model with unknown parameters, an identification equation for the parameters can be acquired. In order to improve the estimation accuracy, the excitation level can be varied to obtain more identification equations than the number of unknown parameters, so that the least-squares criterion can be applied. Details will be given later. While $W_{e}$ requires acceleration, $m$ and $k$, for bounded input and response we have $W_{e} / W_{d} \rightarrow 0$ and $W_{e} / W_{a} \rightarrow 0$ as $T \rightarrow \infty$. Thus for long measured time histories, $W_{e}$ can be neglected.

On the other hand, when $a(t)$ and $x(t)$ are periodic, one can integrate Eq. (2) over a cycle of periodic motion. In that case, the contribution of the conservative components of the oscillator, " $m \ddot{x}+k x$ ", is zero, so that the following energy-balance equation can be obtained

$$
W_{d}=\int_{t}^{t+T} F(x, \dot{x}) \dot{x} d t=\int_{t}^{t+T} a(t) \dot{x} d t=W_{a}
$$

where $T$ represents the period periodic response. Integrating and balancing both sides of equation (3), using measured data and a friction model to express $F(x, \dot{x})$ with unknown parameters, leads to an identification equation for the parameters. The mass and stiffness parameters need not be known for the estimation of damping parameters. 
This paper focuses on the case for which the input is periodic. We will outline the details of the estimation in an example.

\subsection{Base-Excited Oscillator with Coulomb and Viscous Damping}

A schematic diagram showing a base-excited oscillator together with damping components is presented in Figure 1, in which the Coulomb friction and viscous damping are chosen to represent the damping effects existing in an industrial linear-bearing system [37]. The model shown in Figure 1 differs from that of Hundal [17], with the viscous and Coulomb element between the mass and ground instead of parallel with the spring (i.e. between the moving base and the mass). The equation of motion of the oscillator can be written as

$$
m \ddot{x}+c \dot{x}+k x+F_{k} \operatorname{sgn}(\dot{x})=k Y \cos \omega t
$$

where $F_{k} \operatorname{sgn}(\dot{x})$ represents Coulomb friction for pure sliding with no difference between the static and kinetic components, $c$ is the viscous damping coefficient, and $k Y \cos \omega t=a(t)$ represents the harmonic base input.

Applying the left hand side of equation (3) we acquire

$$
W_{a}=\int_{t}^{t+T} a(t) \dot{x} d t=\int_{t}^{t+T} k y \dot{x} d t
$$

which is the energy supplied per cycle, determined by the measured motion signals $y(t)$ and $\dot{x}(t)$ and the stiffness $k$. In addition, the energy dissipated in the dual-damped oscillator during one forcing cycle is

$$
W_{d}=\int_{t}^{t+T} F_{k} \operatorname{sgn}(\dot{x}) \dot{x} d t+\int_{t}^{t+T} c \dot{x} \dot{x} d t=\alpha F_{k}+\beta c,
$$

where $\alpha$ and $\beta$ are defined by the time integrals

$$
\alpha=\int_{t}^{t+T} \operatorname{sgn}(\dot{x}) \dot{x} d t, \quad \beta=\int_{t}^{t+T} \dot{x}^{2} d t .
$$

As such, equation (3), $W_{d}=W_{a}$, leads to

$$
\alpha F_{k}+\beta c=\int_{t}^{t+T} k y \dot{x} d t
$$

Thus, the model of the energy dissipated by the damping forces of the physical system can be balanced against the energy supplied to the oscillator to produce a linear equation in the assumed damping coefficients. Multiple test measurements, $x_{i}(t)$ and $y_{i}(t), i=1, \ldots, n$, will lead to multiple coefficients $\alpha_{i}, \beta_{i}$, and $W_{a i}$, i.e. multiple versions of equation (8), which can be written in matrix form as

$$
\underline{W}=A \underline{p},
$$

where $\underline{W}$ is a vector of elements $W_{a i}, A$ is a matrix for which each row $i$ has the pair of values $\alpha_{i}, \beta_{i}$, and $\underline{p}=\left[F_{k}, c\right]^{T}$ is the vector of unknown parameters. The least-squares solution is

$$
\underline{p}=\left(A^{T} A\right)^{-1} A^{T} \underline{W},
$$


provides an estimate of the parameters, along with a residual $\underline{r}=A \underline{p}-\underline{W}$ that is generally nonzero. The residual can serve as an indicator of the quality of the damping model. In this case, it tells whether " $c \dot{x}+F_{k} \operatorname{sgn}(\dot{x}) "$ can closely represent $F(x, \dot{x})$ occurring at the contact interfaces [38]. Since the coefficients are obtained by integration, high-frequency noise is expected to be filtered, but low frequency noise can have more influence.

Dividing equation (8) by $\alpha_{i}$, we see that a plot of $W_{a i} / \alpha_{i}$ data versus $\beta_{i} / \alpha_{i}$ data is expected to be a straight line, with a slope of $c$ and an intercept of $F_{k}$. (The slope and intercept of the affine least-squares fit of the data in this plot produces a numerically different, and slightly less effective, estimation of the parameters, but allows for a visualization of the linearity of the data.)

\subsection{Harmonic Input and Response}

In the special case when the input is harmonic, and the response is nearly so, it may make sense to make a harmonic approximation to equation (8). Substituting $k y(t)=k Y \cos \omega t, x(t)=X \cos (\omega t-\phi)$, and $\dot{x}(t)=-\omega X \sin (\omega t-\phi)$ into equations (7) and (8), and dividing by $X$, yields

$$
\pi k Y \sin \phi=4 F_{k}+\pi c \omega X
$$

Defining $x_{k}=F_{k} / k, \omega_{n}^{2}=k / m, 2 \xi \omega_{n}=c / m$, and $r=\omega / \omega_{n}$, we can rewrite (11) as

$$
\pi Y \sin \phi=4 x_{k}+2 \pi \xi X r
$$

Equation (11) would be used if the base excitation (or the applied force), $k y$, were known, while more likely Equation (12) would be used if $\omega_{n}$ and $y$ were known. Equation (12) indicates a linear relationship between the input and output amplitudes in which the slope and intercept lead to estimates of the viscous damping factor $\xi$ and the dry-friction parameter $x_{k}$.

The estimation strategy is to generate coefficients of equation (11) or (12) for many input-output cases, perform a least squares fit to a straight line, and obtain the damping parameters from the slope and intercept.

At resonance, $r=1$, and if we assume both $\phi_{1}$ and $\phi_{2}$ are approximately equal to $\pi / 2$, then

$$
\pi Y=4 x_{k}+2 \pi \zeta X
$$

represents a very simple identification equation.

If there are two sets of data, equation (13) can be solved to obtain expressions for $x_{k}$ and $\zeta$. It can be shown that this estimation for $\zeta$ is the same as that of the analytical forced-resonance method [18], developed from analytical response expressions at resonance. In this energy-balance formulation, we have made assumptions of harmonic response with a $90^{\circ}$ phase angle for various input levels at the same excitation frequency. In the forced-resonance method case, we made a "small $\zeta$ " assumption, neglecting $\zeta^{2}$ effects. The equation for $x_{k}$ in the two-point harmonic energy-balance estimation at resonance and that obtained by the two-point analytical forced resonance method (this quantity was denoted as $x_{f}$ in [18]) differ by a factor of $x_{k} / x_{f}=G \pi \xi / 2$, where 


$$
G \cong \frac{\sinh \zeta \pi}{\cosh \zeta \pi-1}
$$

for small $\zeta$. Indeed, as $\zeta \rightarrow 0, G \rightarrow 2 / \pi \xi$. Hence, the $x_{k}$ estimations and the forced-resonance analytical method converge as $\zeta$ decreases. This comment should be taken cautiously, since the Coulomb-only resonant response is unbounded [16]. However, it does suggest that the results for $x_{k}$ should be similar between the analytical scheme and the harmonic energy-balance scheme if $\zeta$ is small.

The major difference between the energy balance and the analytical response methods is that the analytical response is needed in the latter case, but not for the former case. Thus, the energy balance method can be applied to more general systems, such as those with nonlinear stiffnesses, or, for example, quadratic damping laws. The form of equation (8) or (12) depends on the damping model.

\section{NUMERICAL EXAMPLES}

We look at numerical examples using both the general integrated energy balance (IEB) and the harmonic energy balance (HEB) expressions. For comparison with the analytical method [18], we are interested to examine the applicability on and off resonance, and with slip and stick-slip.

\subsection{Slipping Motion}

The parameters corresponding to Equation (4) were $k=100.0 ; m=1.0 ; c=4.0$ and $F_{k}=2.0$. Hence $\zeta=0.2 x_{k}=0.02$. We excited the system at resonance, i.e., $\omega_{1}=\omega_{n}=10.0$, and the excitation levels $Y$ were $0.2,0.4,0.8$, and 1.0. For the harmonic balance, we used the analytical expression presented in Den Hartog's work [16] to obtain the response amplitudes $X=0.43611762,0.93613738,1.93614725$, and 2.43614923. A numerical integration was also monitored to cross check if the pure-sliding motion indeed occurred.

For the harmonic energy balance at resonance, the input-output amplitudes of four responses were processed to form equations (13). A straight line was fit to the $X$, $Y$ data, and from the slope and intercept, we obtained the estimates $\tilde{\xi}=0.19999708$ and $x_{k}=0.020069475$. The mean of the absolute values of residuals in equation (13), normalized by the values of $\pi Y$, was 5.10 e-6. The source of the error includes the assumption that the motion is a single harmonic with an amplitude of den Hartog's peak response solution and a phase of $\pi / 2$, as well as the round-off in the peak response numerals.

To test the IEB method, we used a stiff, low-order ordinary differential equation solver of Bogacki and Shampine (ode23tb) provided in MATLAB (a commercial software) to numerically integrate equations (4) at a constant sampling rate after converting to first-order form [39]. Simpson's rule was used to obtain $W_{a i}, \alpha_{i}$ and $\beta_{i}$ from an arbitrary cycle of the signals.

The least-squares estimation by equation (10) is $\widetilde{\zeta}_{L S}^{\Delta}=0.200002$ and $\widetilde{x}_{k L S}^{\Delta}=0.0199942$. The mean of the absolute values of the residuals (errors in each of equations (9)) normalized by the right hand side of (9), was 8.9 e-6, for numerically integrated noise-free numerical data with a perfect model. The plot of $W_{a i} / \alpha_{i}$ data versus $\beta_{i} / \alpha_{i}$ data was indistinguishable from a straight line.

Although the time-domain output signals are not shown here, they were dominantly sinusoidal. To verify this, we used the fast Fourier transform to check the power spectrum of the output amplitude response. The fundamental frequency differed from the harmonics by more than $120 \mathrm{~dB}$. 
We also tried the off-resonance case. The accuracy of the both integrated and harmonic identification equations was nearly as good as the resonant case, especially the former one. For the same given parameters, the HEB gave $\tilde{\zeta}=0.20851216$ and $x_{k}=0.01199999$. The mean of the absolute values of residuals in equation (13), normalized by the values of $\pi Y$, was $3.24 \mathrm{e}-4$. In contrast with the HEB method, the least-squares from the IEB approach is $\tilde{\zeta}_{L S}^{\Delta}=0.20009491$ and $\tilde{x}_{k L S}^{\Delta}=0.02001505$, with a mean of normalized residual absolute values of $2.67 \mathrm{e}-4$. The method based on den Hartog's analytical solution is more complicated to formulate off resonance, giving the energy balance added appeal over the analytica identification method [18].

\subsection{Stick-Slip Motion}

To induce sticking, we increased the dry friction force and then integrated Eq. (4) directly with $m=1.0$, $F_{k}=8.0, c=4.0, k=100.0$, and $\omega=10.0$. Hence $\omega_{n}=10.0, \zeta=0.2$, and $x_{k}=0.08$. The excitation levels $Y$ were $0.09,0.10,0.11$, and 0.12 , and the response "amplitudes" were $0.002653,0.011098$, 0.025645 , and 0.045310 .

In implementing the harmonic balance identification equation (13), the phase between the excitation and response is needed, for which there is no clear definition since higher harmonics are significant during stick-slip motion. Therefore, we excited the system at resonance and assumed the phase angle to be $\pi / 2$ radians, as pure viscous case at resonance. A line was fit to the $X, Y$ data, whence the slope and intercept produced the estimates $\zeta=0.3403$ and $x_{k}=0.0711$.

The phase issue prompts us to turn to the more general IEB. Because the integration expressions in Equations (3), (5), (6) all involve products of the velocity signal, the integrands become zero when sticking condition occurs.

The least squares estimation corresponding to the stick-slip data gives $\tilde{\xi}=0.202376$ and $\tilde{x}_{k}=0.0798431$. The normalized mean of the absolute values of the normalized residuals was 1.56 e- 4 .

The integrated method is more appropriate in this case since stick-slip responses deviate significantly from the pure-harmonic approximation. An FFT test indicates that the most distorted case corresponding to four different excitation levels has the fundamental frequency $20 \mathrm{~dB}$ larger than other higher harmonics compared to the slip case in which a $120 \mathrm{~dB}$ difference appears.

\section{EXPERIMENTS}

Our experimental system consists of two linear-bearing systems (THK SR20UU) with four linear motion (LM) blocks, an electromagnetic shaker with a power amplifier (Brïel \& Kjær 4809 and 2706), two LVDTs with signal conditioners (Rabinson-Halpern Co., Model 210A-0500), an accelerometer with a charge-type amplifier (Brijel \& Kjær 4371 and 2635), and a data-acquisition system (NI-AT-MIO-16E-10 and LabVIEW). A photograph of the experimental set-up illustrating the linear-bearing system, the electromagnetic shaker, the base-excited plate, the helical springs, the LVDTs, and the accelerometer, is presented in Figure 2.

In Figure 2, the LVDTs were used to sense the displacement signals of the sliding mass and the base excitation, whereas an accelerometer was adopted to check if the response was close to the pure-sliding motion. Mechanical parameters of this experimental rig were $m=1.042 \mathrm{~kg}, k=1568 \mathrm{~N} / \mathrm{m}, \omega_{n}=6.2 \mathrm{~Hz}$. 
The resolution of LVDTs after quantization in the data-acquisition process was about $3 \mu \mathrm{m}$. The dataacquisition system consisted of a PC and the LabVIEW software.

Experimentally, the process required to implement the identification scheme included (a) finding the system's resonance (b) obtaining steady-state responses for various input levels, $Y_{i}$ (c) measuring input and output displacements $x_{i}$ and $y_{i}$, (d) integrating energy coefficients, or extracting displacement amplitudes $X_{i}$ and $Y_{i}$, and (e) seeking the least-squares solution of equation (9) or (13).

Estimates obtained from the energy balance method will be compared to those obtained using the forced-resonance method [18] and the free-vibration decrement method. Finally, in [18], the friction force was measured, and the resulting dry-friction force level (comparable to the $\operatorname{sgn}(\dot{x})$ coefficient $F_{k}$ ) was about $3.6 \mathrm{~N}$, with evidence of some dynamical friction also involved [18,22].

\subsection{Slip Response at Resonance}

First we apply the harmonic approximation. The experimental $\left(Y_{i}, X_{i}\right)$ input and response amplitude data shown in Figure 3 were obtained from the experimental linear-bearing system excited approximately at the resonant frequency [18]. There are totally 100 pairs of input and output amplitudes shown in Figure 3. Every small square shown in this figure indicates one pair of input and output amplitudes, and every input or output amplitude was averaged over more than 10 forcing periods at steady-state [18]. Lines connect the points chronologically as recorded, and thus it seems that the intercept varies slowly, but not the slope (which varies a bit with amplitude), suggesting a slow variation in the dry-friction coefficient.

Recall (Section 3) that the estimates of the viscous parameters corresponding to data shown in Figure 3 are exactly the same for both the HEB and analytical methods at resonance. The estimates of the viscous damping parameters are listed in Table 3. These estimates are obtained by first applying the least-squares fit of equation (13) to the whole data set, the lower $4 / 5,3 / 5$ and $2 / 5$ of the data set. (The reason for doing this is that that $X, Y$ plot seems to depart from a straight line for large amplitudes, suggesting a largeamplitude departure of the model, either in the harmonic assumption e.g. due to nonlinear stiffness, or in unmodeled damping effects [18].) Then we obtained the individual slope and intercept of the linearsquares fits. From these slopes and intercepts, the viscous-damping and dry-friction estimates were obtained accordingly and shown in Table 3.

Next we apply the integrated energy balance. The $\alpha_{i}, \beta_{i}$, and $W_{d i}$ were determined by integrating the experimental signals. The experimental response amplitude actually fluctuated on a small scale. Hence, the integrations were performed over about twelve forcing periods. The input levels were $Y=4.318 \mathrm{~mm}$, $4.910 \mathrm{~mm}, 5.376 \mathrm{~mm}, 5.665 \mathrm{~mm}$, and $6.090 \mathrm{~mm}$, in the range of the lower third of the data for the slip case. The least squares estimation corresponding to the slip data gives $\widetilde{\zeta}=0.103$ and $\widetilde{F}_{k}=4.29 \mathrm{~N}$. Both are very close to the slip estimations based on the entire slip data set, which are slightly low for $\zeta$ and slightly high for $F_{k}$ compared to the free-vibration decrement method and the friction measured in [18]. The mean of the absolute values of normalized residuals was 0.00897 . The data is visually close to the anticipated linear profile (Figure 4).

\subsection{Stick-Slip Response}

We applied the IEB method to a mixed set of stick-slip and pure-sliding response signals. The test conducted consisted of four excitation levels, $Y=2.750 \mathrm{~mm}, 2.625 \mathrm{~mm}, 2.914 \mathrm{~mm}$, and $3.101 \mathrm{~mm}$, at an excitation frequency of $\omega=5.97 \mathrm{~Hz}$, slightly below resonance. Among these excitations, the stick-slip 
case corresponded to the lowest excitation level, whereas the pure-sliding cases corresponded to the other higher excitations. The least squares estimation corresponding to the stick-slip data gives $\tilde{\zeta}=0.0873$ and $\widetilde{F}_{k}=3.23 \mathrm{~N}$, slightly low on both parameters as compared to the slip data and the free-vibration decrement estimation, but still at the right order for a rough approximation. The mean of the absolute values of normalized residuals of equation (9) was 0.0140 .

\section{CONCLUSION}

This paper presents an algorithm for identifying damping information in forced vibration systems. The identification is based on a balance between dissipated energy and energy input. The input energy is obtained from measurements of the input and output displacement. This applied energy per cycle of response is balanced against the dissipated energy, formulated from the damping model. Balancing the measured energy input with the formulated loss leads to parameter identification equations.

The input and output signals are integrated to create numerical coefficients in the identification equations, which are then solved for the parameters in the least-squares sense. If the response is assumed to be harmonic, the input energy is a function of input and output amplitudes, frequency and phase, and the identification equations simplify, omitting the need for integrated coefficients. This, however, is at the cost of accuracy, which shows in some examples.

The harmonic response approximation works very well for the simulated and experimental linear oscillators with Coulomb plus viscous friction in sliding motion. For the simulated case this was successful both on and off resonance. The more general integrated energy method also worked on the numerical and experimental examples with stick-slip responses.

The advantages of the energy balance approach for damping estimation are that it is founded on a simple concept, it is easy to apply, it does not require an analytical solution of the system, it does not require knowledge of the mass and stiffness (except in converting between $\zeta$ and $c$, and $x_{k}$ and $F_{k}$ ), it does not require iterations, and it is theoretically applicable to a class of systems with linear and nonlinear stiffness for which the damping model consists of basis functions and is linear in the coefficients of basis functions. The latter statement was only supported, in this paper, in an example with linear stiffness and Coulomb plus viscous, for which the damping basis functions were $\operatorname{sgn}(\dot{x})$ and $\dot{x}$.

The harmonic energy-balance method described in this paper can be modified slightly to accommodate other friction and damping models. Work is underway on identifying a compliant-contact friction model.

\section{ACKNOWLEDGEMENTS}

Author JWL was supported by the National Science Council (NSC892212E131005) of the Republic of China, Taiwan.

\section{REFERENCES}

[1]. H. L. F. Helmholtz, 1877, On the Sensations of Tone as Physiological Basis for the Theory of Music, translation by A. J. Ellis of Die Lehre von den Tonempfindungen, forth edition; first edition published in 1863, New York: Dover, pp.406. 
[2]. Lord Rayleigh, 1877, The Theory of Sound, Vol. 1, reprinted by Dover, New York, 1945, pp.46-51.

[3]. H. Lorenz, 1924, Lehrbuch der Technischen Physik. Erster Band: Technische Mechanik Starrer Gebilde. Berlin: Verlag von Julius Springer.

[4]. L. S. Jacobsen, R. S. Ayre, Engineering Vibrations, McGraw-Hill, New York, 1958.

[5]. A. Watari, Kikai-rikigaku, Kyouritsu (publisher), 1969.

[6]. B. F. Feeny, J.-W. Liang, A Decrement Method for the Simultaneous Estimation of Coulomb and Viscous Friction, Journal of Sound and Vibration, Vol.195, No.1 (1996), 149-154.

[7]. J.-W. Liang, B. F. Feeny, Identifying Coulomb and Viscous Friction from Free-Vibration Decrements, Nonlinear Dynamics, Vol.16 (1998), 337-347.

[8]. R. Stanway, J. L. Sproston, N. G. Stevens, A note on parameter estimation in nonlinear vibrating systems," Proc. Instn Mech Engrs, Vol 199, no. C1 (1985), 79-84.

[9]. G. Z. Yao, G. Meng, T. Fang, Parameter estimation and damping performance of electrorheological dampers, Journal of Sound and Vibration 204(4) (1997), 575-584.

[10]. Q. Chen, G. R. Tomlinson, Parametric Identification of Systems with Dry Friction and Nonlinear Stiffness Using a Time Series Model, Journal of Vibration and Acoustics 118 (1996), 252-263.

[11]. G. R. Tomlinson, J. H. Hibbert, Identification of the Dynamic Characteristics of a Structure with Coulomb Friction, Journal of Sound and Vibration, 64(2) (1979), 233-242.

[12]. G. R. Tomlinson, An Analysis of the Distortion Effects of Coulomb Damping on the Vector Plots of Lightly Damped System, Journal of Sound and Vibration, 71(3) (1980), 443-451.

[13]. D. V. Iourtchenko, M. F. Dimentberg, In-service identification of nonlinear damping from measrued random vibration, Journal of Sound and Vibration 255(3) (2002), 549-554.

[14]. D. V. Iourtchenko, L. Duval, M. F. Dimentberg, The Damping Identification for Certain SDOF Systems, Proceedings of the SECTAM-XX, Developments in Theoretical and Applied Mechanics, April 16-18, Callaway Gardens, Pine Mountain, GA, (2002), 535-538.

[15]. M. F. Dimentberg, Determination of Nonlinear Damping Function From Forced Vibration Test of a SDOF System, Mechanica Tverdogo Tela, N.2 (1968), 32-34 (in Russian).

[16]. J. P. Den Hartog, Forced Vibration with Combined Coulomb and Viscous Damping, Transactions of the American Society of Mechanical Engineering, Vol.53 (1931), 107-115.

[17]. M. S. Hundal, Response of a Base Excited System with Coulomb and Viscous Friction, Journal of Sound and Vibration, Vol.64 (1979), 371-378.

[18]. J.-W. Liang, B. F. Feeny, Identifying Coulomb and Viscous Friction in Forced Dual-Damped Oscillators, Journal of Vibration and Acoustics 126 (1) (2004), 118-125.

[19]. R. A. Ibrahim, Friction-induced vibration, chatter, squeal, and chaos: part I- mechanics of friction" Friction-Induced Vibration, Chatter, Squeal, and Chaos, ASME Proceedings, DE-Vol. 49 (1992), 107-121.

[20]. B. Armstrong-Hélouvry, P. Dunpont, C. Canudas De Wit, A Survey of Models, Analysis Tools and Compensation Methods for the Control of Machines with Friction, Automatica, 30(7) (1994), 10831138 .

[21]. J. T. Oden, J. A. C. Martins, Models and Computational Methods for Dynamic Friction Phenomena, Comput. Mech. Appl. Mech. Eng., 52(1-3) (1985), 527-634.

[22]. J.-W. Liang, B. F. Feeny, Dynamical friction behavior in a forced oscillator with a compliant contact , Journal of Applied Mechanics, 65(1) (1998), 250-257.

[23]. N. Hinrichs, M. Osetreich, K. Popp, On the Modeling of Friction Oscillator, Journal of Sound and Vibration, Vol.216, No.3 (1998), 435-459.

[24]. D. A. Haessig, B. Friedland, On the modeling and simulation of friction, Journal of Dynamic Systems, Measurement and Control, Vol. 113 (1991), 354-362.

[25]. D. P. Hess, A. Soom, 1990, Friction at a Lubricated Line Contact Operating at Oscillating Sliding Velocities, Journal of Tribology, 112 (1990), 147-152. 
[26]. J. R. Rice, A. L. Ruina, 1983, Stability of Steady Friction Slipping, Journal of Applied Mechanics, 50 (1983), 343-349.

[27]. J. H. Dieterich, Micro-mechanics of Slip Instabilities with Rate- and State-Dependent Friction, volume Fall Meeting Abstract, Volume 324, Eos, Trans., Am. Geophys. Union, 1991.

[28]. A. Ruina, Friction Laws and Instabilities: A Quasistatic Analysis of Some Dry Frictional Behavior, PhD Thesis, Division of Engineering, Brown University, 1980.

[29]. C. Canudas de Wit, H. Olsson, K. J. Astrom, P. Lischinsky, A New Model fro Control of Systems with Friction, IEEE Transactions on Automatic Control 40(3) (1995), 419-425.

[30]. D. M. Tolstoi, 1967, Significance of the Normal Degree of Freedom and Natural Normal Vibrations in Contact Friction, Wear, 10 (1967), 199-213.

[31]. H. Dankowicz, On the Modeling of Dynamic Friction Phenomena, Zeitschrift fuer angewandte Mathematik und Mechanik 79(6) (1999), 399-409.

[32]. A. Harnoy, B. Friedland, B. H. Rachor, Modeling and Simulation of Elastic and Friction Force in Lubricated Bearing for Precise Motion Control, Wear, Vol.172 (1994), 155-165

[33]. A. J. McMillan, A Non-Linear Friction Model for Self-Excited Vibration, Journal of Sound and Vibration, Vol.205, No.3 (1997), 323-335.

[34]. R. V. Kappagantu, B. F. Feeny, Part 1: Dynamical Characterization of a Frictionally Excited Beam, Nonlinear Dynamics, Vol.22, No.4 (2000), 317-333.

[35]. R. V. Kappagantu, B. F. Feeny, Part 2: Proper Orthogonal Modal Modeling of a Frictionally Excited Beam, Nonlinear Dynamics, Vol.23, No.1 (2000),1-11.

[36]. W. T. Thomson, M. D. Dahleh, Theory of Vibration with Applications, Prentice Hall, New Jersey.

[37]. THK, Linear Motion System, THK Co., Ltd., Catalog No.200-1AE, Tokyo, Japan, 1996.

[38]. J. Beck, J., K. Arnold, Parameter Identification in Engineering and Science, John Wiley and Sons, New York, 1977.

[39]. P. Bogacki and L. F. Shampine, A 3(2) pair of Runge-Kutta formulas, Appl. Math. Letters, Vol. 2 (1989) 1-9. 


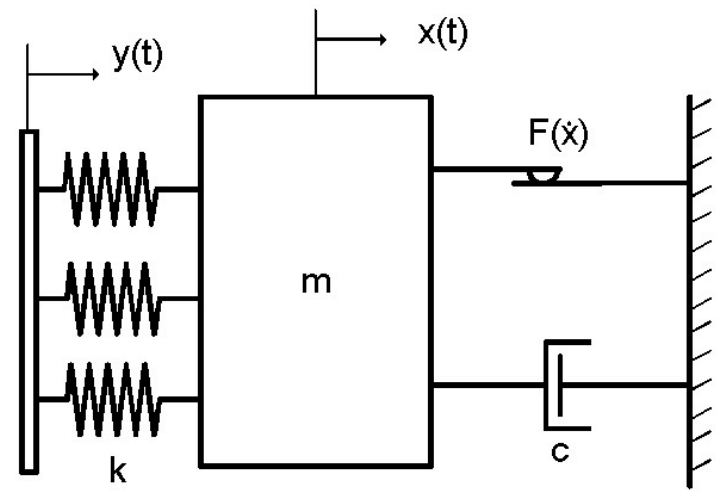

Figure 1: A schematic diagram depicting a single-degree-of-freedom oscillator with viscous, Coulomb friction and base excitation.

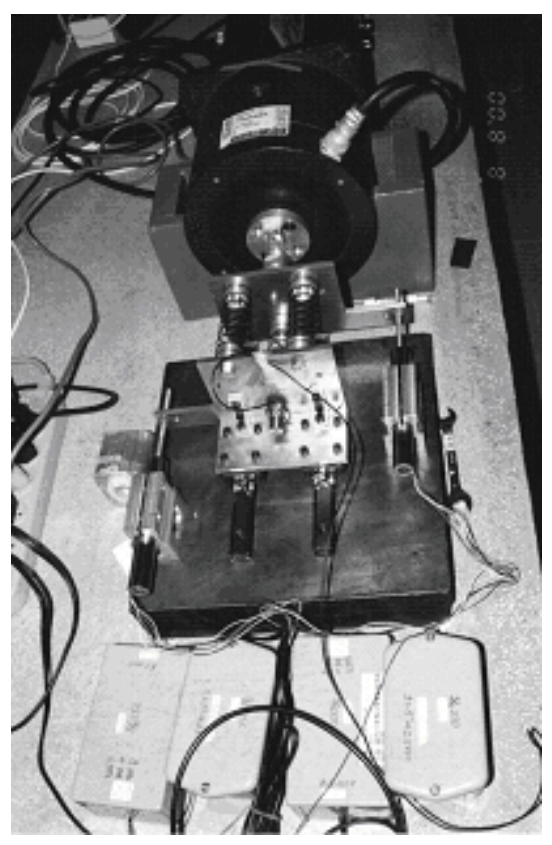

Figure 2: A photograph of the experimental set-up illustrating the linear-bearing systems, the electromagnetic shaker, the LVDTs, etc. 


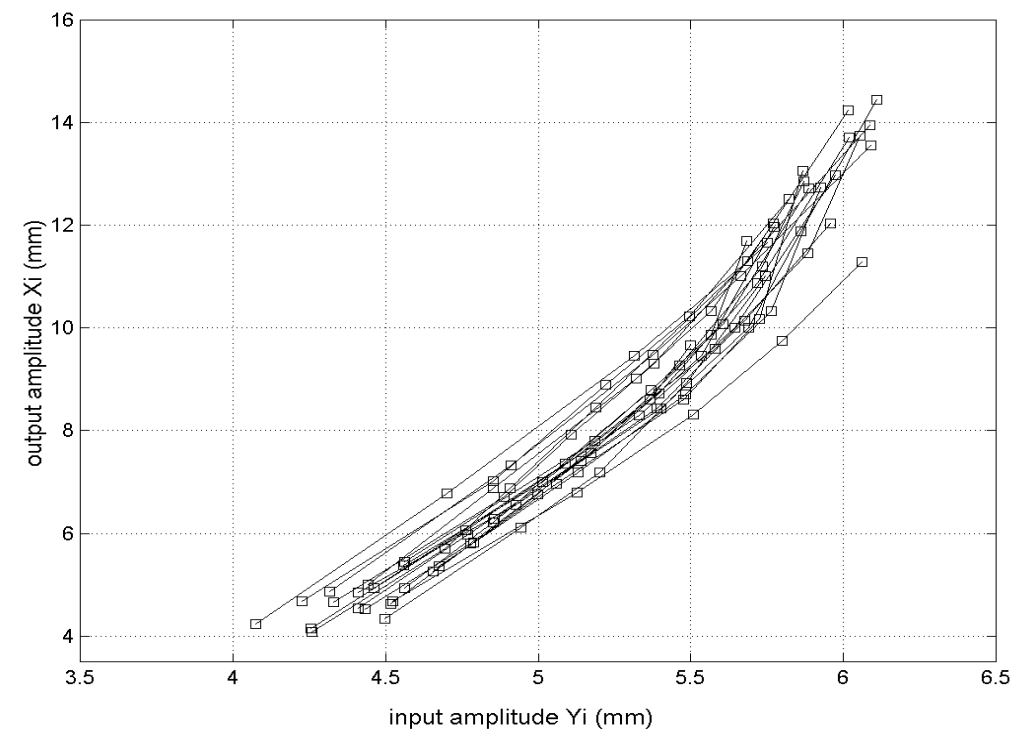

Figure 3: The experimental input-output amplitudes measured nearly at the system's resonance.

Table 3: Experimental estimates of damping parameters of the linear-bearing system.

\begin{tabular}{|l|l|l|l|}
\hline data & $\begin{array}{l}\text { Both methods } \\
\tilde{\zeta}\end{array}$ & $\begin{array}{l}\text { Analytical } \\
\text { method } \widetilde{F}_{o i}\end{array}$ & $\begin{array}{l}\text { Energy } \\
\text { method } \widetilde{F}_{e i}\end{array}$ \\
\hline Whole data set & $\widetilde{\zeta}_{1}=0.0987$ & $\widetilde{F}_{o 1}=4.38$ & $\widetilde{F}_{e 1}=4.38$ \\
\hline $\begin{array}{l}\text { The 4/5 of the } \\
\text { whole data set }\end{array}$ & $\widetilde{\zeta}_{4 / 5}=0.1143$ & $\widetilde{F}_{o 4 / 5}=4.12$ & $\widetilde{F}_{e 4 / 5}=4.13$ \\
\hline $\begin{array}{l}\text { The 3/5 of the } \\
\text { whole data set }\end{array}$ & $\widetilde{\zeta}_{3 / 5}=0.1263$ & $\widetilde{F}_{o 3 / 5}=3.95$ & $\widetilde{F}_{e 3 / 5}=3.96$ \\
\hline $\begin{array}{l}\text { The 2/5 of the } \\
\text { whole data set }\end{array}$ & $\widetilde{\zeta}_{2 / 5}=0.1424$ & $\widetilde{F}_{o 2 / 5}=3.73$ & $\widetilde{F}_{e 2 / 5}=3.75$ \\
\hline $\begin{array}{l}\text { The free- } \\
\text { decrement method }\end{array}$ & $\widetilde{\zeta}_{2 e q}=0.1367$ & $\widetilde{F}_{o 2 e q}=3.52$ & \\
\hline
\end{tabular}




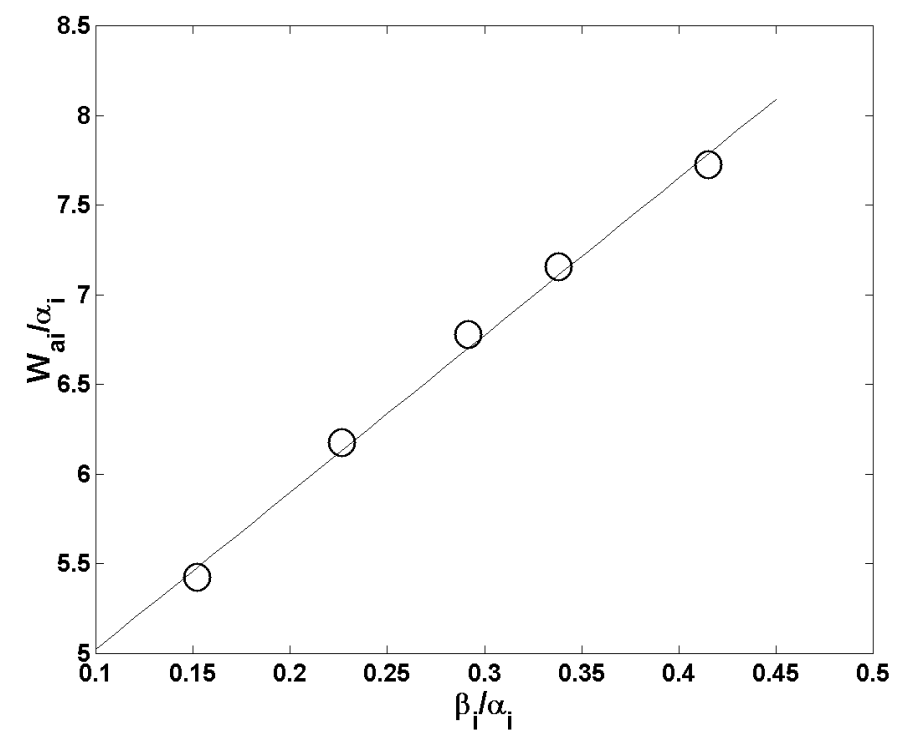

Figure 4. Data distribution of slip response experiments for the integrated energy balance. 